\title{
Quantificação dos impactos da climatização artificial na sensação térmica de transeuntes em termos de alterações no microclima
}

\author{
Quantifying the impacts of air conditioning on the thermal \\ sensation of pedestrians in terms of changes in microclimate
}

Eduardo Krüger ${ }^{[0]}$, Patricia Drach ${ }^{[b]}$

[a] Universidade Tecnológica Federal do Paraná, Curitiba, PR, Brasil

[b] Universidade Federal do Rio de Janeiro, Rio de Janeiro, RJ, Brasil

\section{Resumo}

O presente estudo analisa efeitos do uso de ar-condicionado na sensação térmica devido a interferências na aclimatação de indivíduos no espaço aberto, a partir de estudo de campo no centro do Rio de Janeiro. Campanhas de monitoramento foram realizadas em períodos quentes entre 2012 e 2015, com uso de estação meteorológica concomitantemente à aplicação de questionários estruturados segundo a ISO 10551, aos quais se incluíram questões acerca do uso de ar-condicionado. 0 índice de conforto utilizado para interpretar dados microclimáticos foi o UTCI (Universal Thermal Climate Index). Dos resultados obtidos, notou-se que o uso frequente de ar-condicionado impacta a percepção térmica dos usuários, interferindo na definição de limites de conforto. Verificou-se uma queda de $2^{\circ} \mathrm{C}$ em unidades UTCI para uma situação de início de estresse térmico por calor para o grupo menos aclimatado (usuários habituais de ambientes climatizados artificialmente), fato que sugere redução na tolerância térmica ao calor dessa população. A partir dos resultados encontrados e para o desenvolvimento da área de conforto em espaços abertos no Brasil (cuja maior parte do território apresenta clima tropical), no que tange a levantamentos de percepção térmica junto à população, recomenda-se a introdução do questionamento acerca do uso de ar-condicionado.

Palavras-chave: Microclima urbano. Climatização artificial. Conforto em espaços abertos.

EK é Engenheiro civil, Doutor em Arquitetura - Leiniz Universität Hannover, e-mail: ekruger@utfpr.edu.br PD é Arquiteta e Urbanista, Doutora em Modelagem Computacional, e-mail: patricia.drach@gmail.com 


\section{Abstract}

The study analyzes the effects of air-conditioning (AC) usage on thermal sensation due to interferences in acclimatization in outdoor spaces, from field data in Rio de Janeiro. Field monitoring campaigns have been carried out in warm periods between 2012 and 2015, with microclimate monitoring and concurrent comfort surveys according to ISO 10551, to which questions on the use of AC were added. The thermal index employed for interpreting microclimatic data was UTCI (Universal Thermal Climate Index). Results showed that the frequent use of AC could have an impact on the thermal perception of passersby, thus confounding the definition of thermal comfort limits. A drop of $2^{\circ} \mathrm{C}$ was estimated in terms of UTCI units for the onset of heat thermal stress for the less acclimatized group (typical users of air-conditioned spaces), which suggests a reduction in thermal tolerance to heat in such population stratum. From results obtained and towards the development of the field area outdoor thermal comfort in Brazil (with mostly tropical climate conditions) it is recommended the introduction of questioning as regards time of residency in outdoor comfort surveys.

Keywords: Urban microclimate. Air conditioning. Outdoor thermal comfort.

\section{Introdução}

O interesse principal do tema abordado está relacionado à necessidade de se definirem estratégias de desenho urbano climaticamente orientado de forma a mitigar o desconforto térmico no meio urbano. 0 estudo insere-se em uma pesquisa mais abrangente, que analisa a percepção térmica de indivíduos em espaços abertos a partir de equipamentos de medição e protocolos de levantamento de dados seguindo uma mesma padronização (Johansson et al., 2014). 0 efeito de variáveis biométricas dos indivíduos entrevistados (Krüger e Rossi, 2011; Krüger e Drach., 2017a; Krüger e Drach., 2017b), a influência da conformação urbana (Minella et al., 2011; Krüger et al., 2017a) assim como a calibração de índices de conforto térmico no espaço aberto (Rossi et al.,2012; Krüger et al., 2017b) foram investigados durante diferentes etapas da pesquisa.

No estudo tratado neste artigo, a partir de dados medidos e de respostas térmicas de transeuntes, procurou-se levantar a influência de hábitos individuais em relação ao uso de climatização artificial na percepção térmica reportada pelos entrevistados. Isso levando-se em conta que, para as condições climáticas do Rio de Janeiro, o principal desafio de um planejamento urbano climaticamente responsável é minimizar por meio de atributos urbanos (morfologia, vegetação urbana, fontes d'água, proximidade à orla) o estresse térmico devido ao calor, condição típica do clima local. Estudos realizados para a cidade do Rio de Janeiro vêm apontando a possibilidade de interferir na sensação térmica dos usuários dos espaços abertos através de alterações na morfologia urbana, tais como a modificação da forma, da posição, dos materiais, dentre outros (Barbosa et al., 2010a, 2010b; Drach \& Corbella, 2010). Assim, a introdução ou retirada de equipamentos urbanos, emprego de vegetação e escolha adequada de materiais de revestimento podem ser intervenções passíveis de minimizar o problema do sobreaquecimento urbano.

A determinação de zonas de conforto a partir de dados de percepção térmica de usuários de espaços abertos é fundamental para balizar os objetivos a serem atingidos com o desenho urbano climaticamente responsável. Tal tarefa, porém, envolve tanto aspectos fisiológicos da sensação térmica quanto fatores psicológicos e subjetivos, os quais desafiam o pesquisador na definição de limites de estresse térmico. Um aspecto que traz complexidade a essa definição é o histórico térmico dos indivíduos aos quais são aplicados os questionários de percepção térmica. Um artigo seminal nesse sentido mostra a influência do histórico térmico em grupos de indivíduos que frequentavam ambientes climatizados ou ambientes naturalmente ventilados em situação de calor na Tailândia (Busch, 1992). As diferenças observadas no limite superior de conforto, expressas em termos de temperatura do ar, chegaram a $3^{\circ} \mathrm{C}$ 
entre ambos os grupos, com os indivíduos sujeitos à climatização artificial mostrando menor tolerância ao calor.

Chun et al. (2008), Cândido et al. (2010), De Vecchi et al. (2012), Fadeyi (2014) realizaram pesquisas independentes com grupos de indivíduos os quais apresentavam diferentes históricos térmicos. Tais autores observaram tolerância reduzida à condição de calor por pessoas que usualmente frequentam ambientes climatizados.

Em uma outra linha, oposta àquela, alguns estudos avaliaram se mudanças súbitas no ritmo habitual às condições climáticas, como durante episódios de onda de calor, alteram a percepção térmica de pessoas expostas a espaços abertos. 0 estudo realizado por Lam et al. (2016), por exemplo, analisa a reação de australianos durante uma onda de calor em parques, utilizando o índice de conforto em espaços abertos"Universal Thermal Climate Index" (UTCI) como referência para análise de votos de percepção térmica durante e após uma onda de calor ocorrida em Melbourne. Verificou-se que os entrevistados apresentaram maior percentual de votos de sensação de calor durante a onda de calor, para uma mesma faixa de UTCI verificada logo após sua passagem, levando os autores a concluir que o fator expectativa térmica afetou a percepção de estresse/conforto da amostra.

Conforme apontado por Chen \& Ng (2012), a percepção térmica em espaços abertos é assunto complexo, o qual pode ser subdividido em diversos níveis de preocupação. Em trabalhos de campo, o estímulo ambiental é, em geral, o principal aspecto a ser avaliado, o qual é representado de forma objetiva e estática pelas variáveis microclimáticas monitoradas no momento da entrevista. Os questionários obtidos, porém são dinâmicos e subjetivos. Dinâmicos, pois a adaptação a condições ambientais é progressiva (aclimatação de curto prazo) e afetada também pelo histórico térmico do entrevistado (conforme Chunet al., 2008; Cândido et al., 2010; DeVecchi et al., 2012; Fadeyi, 2014) ou, conforme visto, pela expectativa térmica sazonal ou episódica, como durante a ocorrência de ondas de calor. Subjetivos, pois a resposta obtida em termos de sensação térmica experimentada não é sempre consistente com os dados objetivos monitorados. Chen \& Ng (2012) advogam que o uso de espaços externos não se deve apenas ao estado do corpo ("stateofbody"), mas também ao estado mental ("stateofmind") do indivíduo, apontando para a necessidade de se desenvolverem ferramentas de avaliação que levem em conta ao menos os quatro níveis seguintes: aspectos físicos, fisiológicos, psicológicos e sociais/comportamentais.

Em ambientes internos climatizados, a redução na tolerância térmica por calor é compensada com aumento na demanda por climatização artificial. Em contraste, em espaços externos, a consequência é o aumento do estresse térmico por calor no verão, o que pode traduzir em um aumento na pressão por parte de usuários dos espaços externos por projetos urbanísticos de mitigação do calor.

Arens et al. (2010) ressaltam o papel da redução de consumo energético em climatização artificial em ambientes internos, examinando o desempenho em termos de níveis de conforto reportado por usuários de edificações classes 'A' (controle térmico mais rigoroso, com, por exemplo, menor variação do índice PMV interno), ' $\mathrm{B}$ ', 'C' (ambas menos rigorosas), conforme a ASHRAE 55. A análise estatística de resultados de estudos realizados em edificações climatizadas, disponíveis em bancos de dados com aceitabilidade térmica dos ocupantes, mostrou que ambientes com controle térmico rigoroso (do tipo A) não trazem necessariamente maior grau de aceitabilidade térmica que ambientes to tipo $\mathrm{B}$ e $\mathrm{C}$, o que corrobora a hipótese do 'conforto adaptativo'. Luoet al. (2016) formulam a seguinte questão: seria de se esperar de indivíduos que ocupam ambientes considerados termicamente ideais por longos períodos se tornem tão exigentes ("fussy") com o seu ambiente térmico de modo que não há acréscimos, e podendo até mesmo haver decréscimos, em sua satisfação térmica com o ambiente?

Partindo da ideia de que a frequência no uso de ambientes climatizados perturba a percepção térmica de indivíduos, uma vez que estes estejam expostos a condições externas não controladas e com maior variabilidade térmica que em ambientes internos, o artigo tem como foco a análise da relação entre o histórico térmico dos entrevistados e sua percepção térmica (votos de sensação térmica) no espaço aberto. 0 objetivo do estudo foi de traduzir em termos quantitativos (variáveis meteorológicas que compõem o índice de conforto em espaços abertos adotado como parâmetro de análise),via avaliação paramétrica, alterações verificadas nos 
votos de sensação térmica pelo uso frequente ou não de ambientes condicionados pelos indivíduos entrevistados.

\section{Metodologia}

Conforme descrito em pormenores por Krüger et al. (2015), a pesquisa de campo foi realizada em ruas centrais do Rio de Janeiro, abrangendo uma amostra de pessoas que trabalha e circula no Centro da cidade do Rio de Janeiro e reside na área do Grande Rio e Grande Niterói, dadas as semelhanças climáticas das duas regiões. 0 levantamento de dados foi realizado a partir de questionários estruturados de acordo com a ISO 10551 (ISO, 1995) acerca da percepção térmica ao ar livre, aplicados à população local. Os questionários foram aplicados concomitantemente a medições microclimáticas em áreas de pedestre com estação meteorológica completa Davis Vantage Pro2, acrescida de um termômetro de globo, para o cálculo da temperatura radiante média, segundo procedimento e equacionamento constantes da norma ISO 7726 (ISO, 1998), para convecção forçada. A análise dos dados se utiliza de estatística descritiva, análise de regressão e correlacional. As diferenças entre séries de dados foram verificadas adicionalmente quanto ao seu significado estatístico por meio dos testes $\mathrm{F}$ e $\mathrm{t}$.

Área de estudo e locação dos pontos de medição

De acordo com a classificação de Koeppen-Geiger (Peelet al., 2007), a cidade do Rio de Janeiro (22 $54^{\prime}$ $10^{\prime \prime} \mathrm{S}, 43^{\circ} 12^{\prime} 27^{\prime \prime} \mathrm{W}$ ) é caracterizada por um clima tropical com chuvas de verão (Aw). Todos os pontos selecionados, nos quais foram efetuadas as medições e entrevistas a transeuntes, se localizam em ruas de pedestre com acesso a veículos permitido apenas para serviços (entregas e retiradas de mercadorias e outras atividades relacionadas à manutenção do comércio, escritórios, empresas etc.). A seleção dos pontos levou em conta o fato de os locais serem representativos para as avaliações, com diferenças na morfologia urbana capazes de representar a diversidade observada em um centro urbano, totalizando os sete pontos de monitoramento conforme apresentado na Figura 1.
Ressalta-se, porém, que, para o objetivo deste artigo, a influência da geometria urbana na percepção térmica ao nível do pedestre não foi considerada.

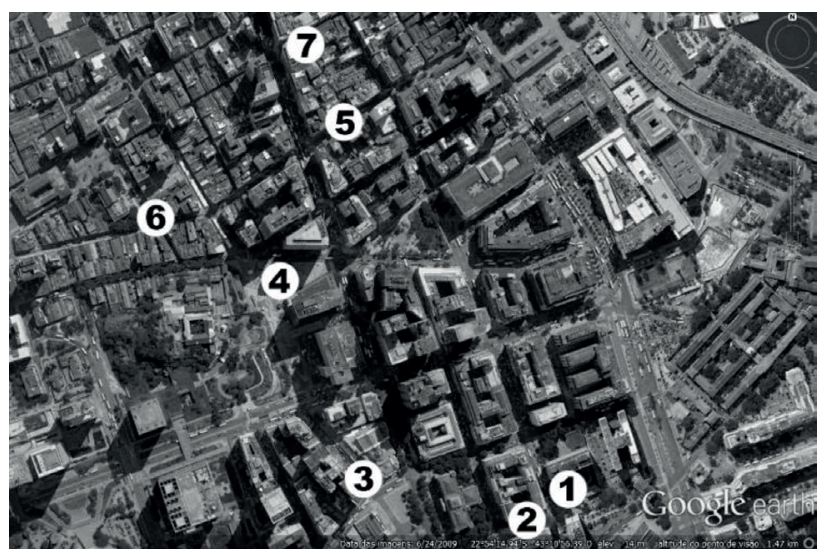

Figura 1 - Mapa com o trecho de pedestres analisado e os sete pontos de medição.

Fonte: Google Earth, gerado a partir de imagem de 06/04/2009.

\section{Coleta de dados físicos e subjetivos}

A coleta de dados físicos foi realizada com uma estação meteorológica Davis Vantage Pro2, equipamento wireless constituído por duas partes: conjunto de sensores; e console digital (datalogger) responsável pelo armazenamento de dados. 0 conjunto de sensores é composto de sensor de temperatura e umidade do ar (Ta/UR), anemômetro de copo com pá de vento (medição da velocidade do ar, v), piranômetro de silício e coletor de água de chuva. Para a obtenção da temperatura radiante média (TRM), adotou-se uma esfera de PVC com diâmetro aproximado de $110 \mathrm{~mm}$, pintada na cor cinza-médio, na qual se inseriu um medidor de temperatura (Tinytag TGP-4500). 0 posicionamento do conjunto de sensores atendeu às recomendações da ISO 7726 (ISO, 1998). Utilizaram-se sensores de temperatura do ar e de umidade relativa, e o termômetro de globo, fixados à altura de $1,10 \mathrm{~m}$. 0 piranômetro foi fixado a 1,40 m, e o anemômetro, a 1,50 m, de modo que os dados coletados estivessem o mais próximo possível do nível do pedestre/entrevistado. A temperatura radiante média (TRM) foi calculada a partir dos dados de temperatura (sensor encapsulado), temperatura do ar e da velocidade do ar medidos em cada ponto, sendo utilizada a equação para convecção forçada, de acordo com a ISO 7726 (ISO, 1998). A emissividade da esfera de PVC cinza foi assumida 
como 0,95. A gravação dos dados ocorreu a cada $5 \mathrm{~s}$, sendo a média composta para cada minuto.

0 período durante o qual ocorreram as entrevistas, concomitantemente às medições microclimáticas foi das $10 \mathrm{hs}$ às $15 \mathrm{hs}$. 0 questionário foi definido a partir da ISO 10551 (ISO, 1995), e entre as questões foram coletados dados biométricos, relacionados a idade, gênero e índice de massa corporal dos indivíduos, os quais foram analisados em outro estudopor Krüger e Drach. (2017b), questões relacionadas ao processo de aclimatação a longo e a curto prazos (tempo de residência no Rio de Janeiro ou imediações, uso ou não de ar-condicionado em casa ou no trabalho), as quais foram tratadas em parte neste artigo, e informações gerais acerca da vestimenta utilizada, adotando-se uma tabela-resumo de trajes típicos, conforme apresentada no Anexo A da ISO 9920 (ISO, 2007). Para avaliar a percepção térmica (sensação e preferência) foi utilizada a escala simétrica de sete pontos. No caso da sensação térmica ("Como você se sente neste preciso momento?"), as respostas foram categorizadas em: (-3) "muito frio"; $(-2)$ "frio"; (-1) "pouco frio"; (0) "neutro"; $(+1)$ "pouco calor"; (+2) "calor"; e (+3) "muito calor".

No caso da taxa metabólica, foi assumido um valor fixo de 2,3 Met, o que corresponde a pessoa caminhando a uma velocidade de $4 \mathrm{~km} / \mathrm{h}$, situação típica no caso de passantes.

As variáveis tempo de residência e tempo de permanência no espaço aberto foram consideradas como critério de exclusão, quando inferiores a 6 meses e 15 min respectivamente. 0 limite de 15 min foi apropriado do mínimo recomendado pela ASHRAE 55 (ASHRAE, 2004) para estudos de conforto em espaços internos. Foram excluídas pessoas com pesos extremos (máximo e mínimo) e crianças.

A amostra foi de 985 indivíduos, sendo 61\% entrevistados do sexo masculino e $39 \%$ do sexo feminino, com idade variando entre 13 e 87 anos. Quanto às variações climáticas encontradas ao longo dos diversos dias de medição, a Tabela 1 mostra as faixas monitoradas.

Tabela 1 - Faixas de variação dos dias monitorados

\begin{tabular}{|c|c|c|c|c|c|}
\hline & $\begin{array}{l}\text { Temperatura do } \\
\operatorname{ar}\left(\mathrm{T}_{\mathrm{a}} \mathrm{em}^{\circ} \mathrm{C}\right)\end{array}$ & $\begin{array}{l}\text { Umidade relativa } \\
\text { (UR em \%) }\end{array}$ & $\begin{array}{l}\text { Velocidade do ar } \\
\text { ( } v \text { em } \mathrm{m} / \mathrm{s} \text { ) }\end{array}$ & $\begin{array}{l}\text { Irradiância solar global e } \\
\text { refletida (lg em W/m²) }\end{array}$ & $\begin{array}{l}\text { Temperatura Radiante } \\
\text { média (TRM em }{ }^{\circ} \mathrm{C} \text { ) }\end{array}$ \\
\hline $\begin{array}{l}\text { Faixa de } \\
\text { variaç̃ão }\end{array}$ & $24,9-36,8$ & $37-82$ & $0-2,7$ & $21-1218$ & $13,1-76,4$ \\
\hline
\end{tabular}

Fonte: Autores

Índice térmico adotado

Utilizou-se um índice térmico para espaços abertos de modo a integrar as diversas variáveis microclimáticas medidas nos pontos de monitoramento. 0 Universal Thermal Climate Index (UTCI) foi elaborado com base no modelo termorregulatório multinodal desenvolvido por Fiala et al. (2012) e é baseado em uma temperatura equivalente em ambiente de referência. As variáveis de entrada para o cálculo do UTCI provêm dos levantamentos in loco. No UTCI, a vestimenta é estimada segundo modelo de isolamento térmico da vestimenta embutido no próprio índice.

No Brasil já há alguns trabalhos que utilizam o índice UTCI para a predição da sensação térmica no espaço aberto (Rossiet al., 2012; Monteiro\& Alucci,
2012; Ninceet al., 2013). 0 índice UTCI foi calculado utilizando-se o software BioKlima v. 2.6, fazendo-se a devida correção dos dados de vento para $10 \mathrm{~m}$ de altura, conforme os procedimentos de cálculo desseíndice (Brödeet al., 2012).

\section{Resultados encontrados}

Os votos de sensação térmica declarados pelos transeuntes se encontram, em sua maior parte, em situação de desconforto térmico por calor (votos de 1 a 3), totalizando $74 \%$ dos votos totais. 0 fator vestimenta foi considerado como de baixo impacto na sensação térmica, uma vez que a variação verificada em termos de isolamento térmico foi de 0,2-0,7 clo $\left(0,03-0,11 \mathrm{~m}^{2} . \mathrm{K} / \mathrm{W}\right)$, o que corresponde a vestimentas leves de verão. 
A pergunta do questionário aplicado referente à utilização de equipamentos de condicionamento de ar em casa ou no ambiente de trabalho permite a análise de seus reflexos nos votos de sensação térmica informados pelos entrevistados. Embora a comparação seja por agrupamento de situações, e não pela exposição dos indivíduos a condições diversas de permanência em ambientes climatizados (em um estudo experimental em câmara climática, por exemplo), pode-se notar uma tendência a um maior desconforto térmico por calor nos grupos de entrevistados que reportaram ter acesso parcial ou com maior frequência a ambientes climatizados.

A Tabela 2 apresenta o valor médio obtido por grupo para a sensação térmica, seu correspondente desvio padrão (com o coeficiente de variação CV = desvio padrão/média, em percentual) e as diferenças relativas para a situação-base, sem uso de ar-condicionado (dados da segunda coluna).

Tabela 2 - Comparações agrupadas de votos de sensação térmica vis-à-vis uso de equipamento de ar-condicionado em casa e no trabalho

\begin{tabular}{|c|c|c|c|c|c|}
\hline & Sem AC & $\begin{array}{c}\text { AC em casa OU no } \\
\text { trabalho }\end{array}$ & AC em casa & AC no trabalho & $\begin{array}{c}\text { AC em casa E no } \\
\text { trabalho }\end{array}$ \\
\hline $\begin{array}{l}\text { Sensacuão térmica } \\
\text { (média dos votos) }\end{array}$ & 1,2 & 1,4 & 1,6 & 1,3 & 1,6 \\
\hline Sensação térmica (dp e CV) & $1,1 / 93 \%$ & $1,2 / 79 \%$ & $1,2 / 74 \%$ & $1,1 / 83 \%$ & $1,1 / 68 \%$ \\
\hline $\mathrm{n}^{\mathrm{n}}$ (após procedimento de geração de bins) & $\begin{array}{l}282 \\
276\end{array}$ & 387 & 164 & 223 & $\begin{array}{l}316 \\
301\end{array}$ \\
\hline Diferenças (Sensaç̃õo térmica) & - & 0,2 & 0,3 & $0,1^{*}$ & 0,4 \\
\hline
\end{tabular}

*Diferença não significativa para um nível de confiança de $95 \%$.

Fonte: Autores

Os resultados obtidos em termos de sensação térmica são comparados aos valores calculados na escala UTCI a partir dos dados meteorológicos, para duas condições: para o grupo de pessoas que utilizam constantemente ar-condicionado (em casa e/ ou no trabalho) e para aqueles que não o utilizam. A diferença em termos de aumento do desconforto por calor chegou a 0,4 voto de sensação térmica. 0 impacto do uso do ar-condicionado mostra-se de forma consistente nos resultados encontrados: quanto mais frequente o uso (ou disponibilidade) de climatização artificial, conforme reportado pelo entrevistado, maior o desconforto por calor no espaço aberto, com exceção da situação do uso de ar- condicionado no ambiente de trabalho, a qual não acarretou em diferença significativa.

Adotou-se o agrupamento de respostas de sensação térmica para cada variação de um grau na escala UTCI (dados agrupados por "bin" de UTCI), conforme procedimento sugerido por De Dear \& Fountain (1994) e utilizado em estudos semelhantes (Deuble\& De Dear, 2012; Krügeret al., 2012; Linet al., 2013; Ndetto\& Matzarakis, 2013; De Dearet al., 2014; Ndetto\& Matzarakis, 2015). A partir dos dados agrupados, gerou-se uma linha de tendência linear para cada série (Figura 2).

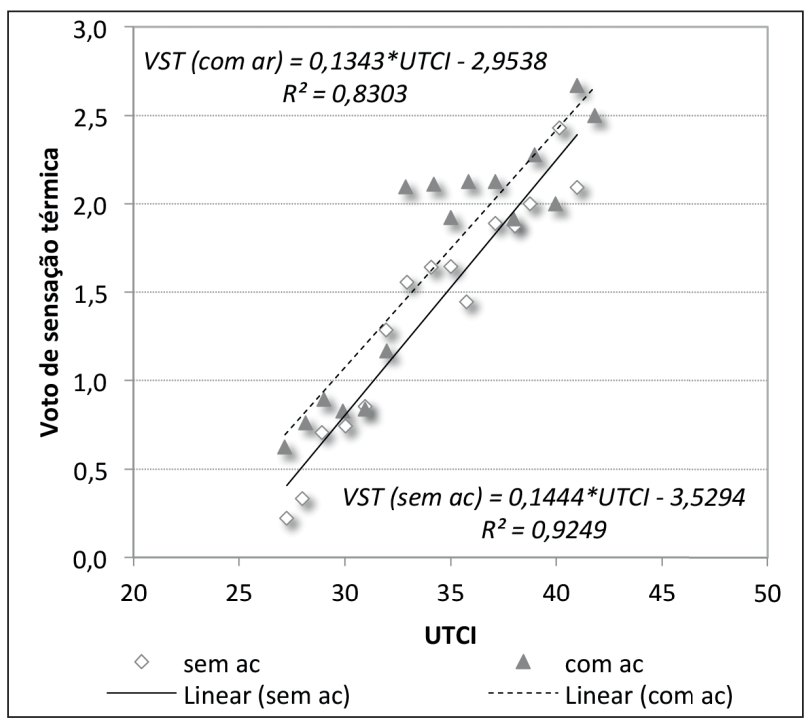

Figura 2 - Sensação térmica média versus valores encontrados na escala UTCI (valores agrupados a cada grau na escala UTCI) - comparação entre grupo aclimatado e grupo que utiliza com frequência climatização artificicial, conforme reportado pelos transeuntes

Fonte: Autores. 
Foram geradas duas equações de regressão para a obtenção da sensação térmica estimada a partir de valores na escala UTCI, para ambos os grupos: grupo aclimatado (que não reportou uso de ar-condicionado) e grupo de pessoas que utilizam constantemente ar-condicionado (em casa e no trabalho). A correlação encontrada foi elevada, mostrando a aplicabilidade do índice UTCI para os dois subgrupos da amostra. Os dados de campo (agrupados em bins) sofreram cortes ( $\mathrm{n}=276$ e $\mathrm{n}=301$, grupo aclimatado e grupo que usa climatização artificial com frequência, respectivamente) para que cada bin, ou variação de $1{ }^{\circ} \mathrm{C}$ na escala UTCI, contivesse a média de no mínimo 5 votos de sensação térmica (comparar com Tabela 2). A faixa de variação de temperatura na escala UTCI foi de $27-42^{\circ} \mathrm{C}$. A temperatura neutra encontrada nos dois casos foi de $24,4^{\circ} \mathrm{C}$ e $22,0^{\circ} \mathrm{C}$ (escala UTCI) para a situação neutra (voto 0 ) sem uso e com uso de ar-condicionado respectivamente. Existe uma semelhança nos coeficientes angulares das equações de tendência das duas curvas. Nota-se a necessidade de se reduzir o valor UTCI em $2,4^{\circ} \mathrm{C}$ para garantia de conforto, relativamente ao caso-base, quando há uso frequente de ar-condicionado.
Para a situação inicial de calor, $(+1)$ "pouco calor", as temperaturas na escala UTCI diferem em cerca de $2,0^{\circ} \mathrm{C}$ na escala UTCI, sendo $31,4^{\circ} \mathrm{C}$ e $29,4^{\circ} \mathrm{C}$ para os grupos sem uso e com uso frequente de ar-condicionado respectivamente.

\section{Análise paramétrica dos efeitos}

microclimáticos no cálculo do UTCI

Para efeito de comparação, realizou-se um procedimento paramétrico de forma a se avaliar a diferença relativa encontrada em média para o UTCI (para $\mathrm{n}=985$ ), quando se alteram individualmente as variáveis microclimáticas relevantes para este cálculo. A Tabela 3 apresenta variações no UTCI médio encontrado em função de alterações discretas nas variáveis Ta, UR, v e TRM, caso a caso.Procurou-se desta forma avaliar qual incremento em termos de estresse térmico seria necessário para cada variável analisada de modo a se obter semelhante incremento na temperatura inicial de estresse térmico para o grupo que reportou uso frequente de ambientes climatizados (conforme apresentado na seção anterior).

Tabela 3 - Análise paramétrica de alterações individuais nas variáveis microclimáticas na obtenção de valor no índice UTCl considerando a totalidade de dados registrados

\begin{tabular}{clcc}
\hline Variável & Alteração realizada & Valor médio do UTCl $\left({ }^{\circ} \mathrm{C}\right)$ & $\begin{array}{c}\text { Diferençá relativa ao valor médio para a } \\
\text { série original agrupada }\left({ }^{\circ} \mathrm{C}\right)\end{array}$ \\
\hline Ta $\left(^{\circ} \mathrm{C}\right)$ & soma $0,5^{\circ} \mathrm{C}$ & 33,8 & 0,5 \\
& soma $1{ }^{\circ} \mathrm{C}$ & 34,2 & 0,9 \\
& soma $1,5^{\circ} \mathrm{C}$ & 34,8 & 1,5 \\
& soma $2^{\circ} \mathrm{C}$ & 35,3 & 2,0 \\
\hline UR (\%) & soma $5 \%$ & 33,7 & 0,5 \\
& soma $10 \%$ & 34,2 & 1,0 \\
& soma $20 \%$ & 35,5 & 2,2 \\
\hline $\mathrm{V}(\mathrm{m} / \mathrm{s})$ & subtrai $0,5 \mathrm{~m} / \mathrm{s}$ & 33,5 & 0,3 \\
& subtrai $1 \mathrm{~m} / \mathrm{s}$ & 33,8 & 0,5 \\
& ar parado & 33,8 & 0,5 \\
\hline TRM $\left({ }^{\circ} \mathrm{C}\right)$ & soma $5^{\circ} \mathrm{C}$ & 34,5 & 1,2 \\
& soma $7,5^{\circ} \mathrm{C}$ & 35,1 & 1,9 \\
& soma $10^{\circ} \mathrm{C}$ & 35,8 & 2,5 \\
& soma $15^{\circ} \mathrm{C}$ & 37,0 & 3,7 \\
\hline
\end{tabular}

Fonte: Autores. 
Na Tabela 3, os resultados em negrito foram os que mais se aproximaram do acréscimo em termos de UTCI para o grupo menos aclimatado (que reportou uso frequente de ambientes condicionados). Desta forma, os resultados mostraram ser necessário um incremento na temperatura do ar de $2,0^{\circ} \mathrm{C}$ no cálculo do UTCI (série completa), de modo que a alteração média no valor do índice seja da mesma ordem de grandeza que a diferença encontrada entre os dois grupos de indivíduos na definição de início de estresse térmico por calor. Quanto às demais variáveis, o incremento necessário da umidade relativa seria de cerca de $20 \%$, e da temperatura radiante média em torno de $7,5^{\circ} \mathrm{C}$. No caso do vento, mesmo simulando-se uma condição de ar estagnado $(\mathrm{v}=0 \mathrm{~m} / \mathrm{s})$, a redução no UTCI seria de apenas $0,5^{\circ} \mathrm{C}$. Ou seja, mesmo anulando-se os movimentos de ar no plano do pedestre, o impacto no UTCI calculado para essa situação face ao caso base seria ainda bem inferior à diferença encontrada nas temperaturas do índice definidoras do início de estresse por calor entre os grupos aclimatado e usuários contumazes de climatização artificial, o que nos dá uma ideia do impacto do uso continuado da climatização na sensação térmica de indivíduos no espaço aberto.

Os gráficos mostram o efeito individual de alterações discretas nas variáveis relevantes para o cálculo do UTCI (Figura 3), a partir do qual se montou a Tabela 3.

a)

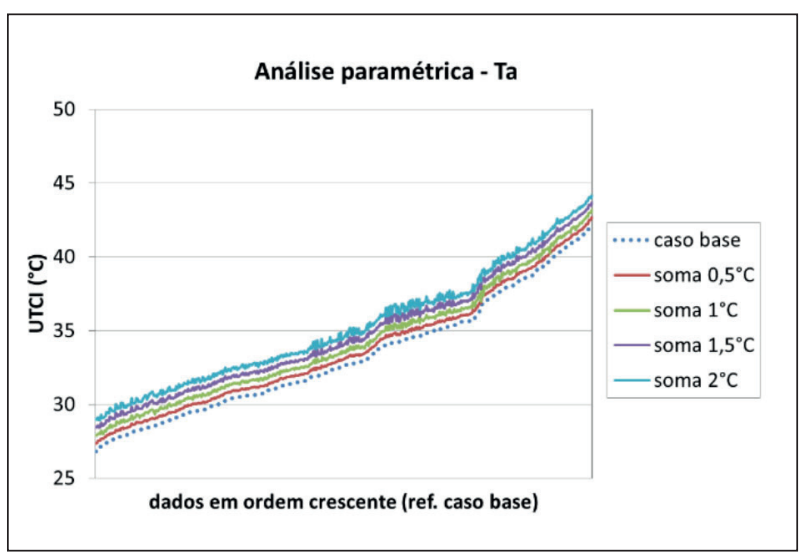

b)

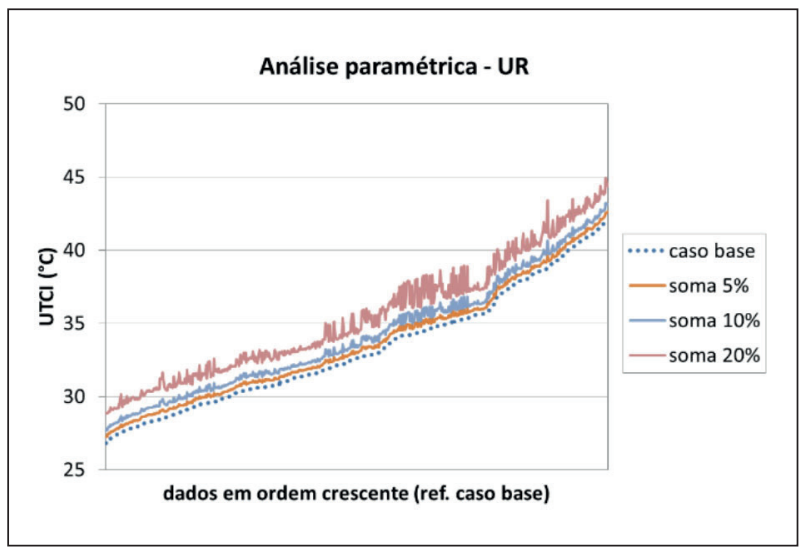

c)

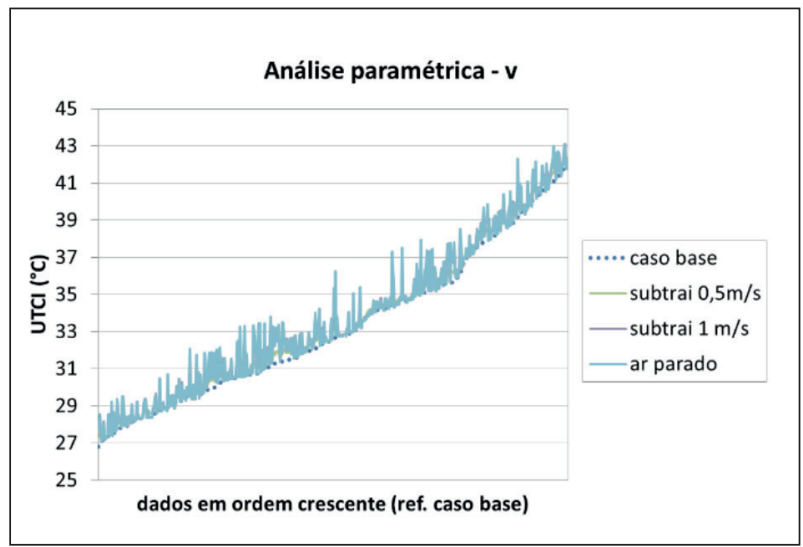

d)

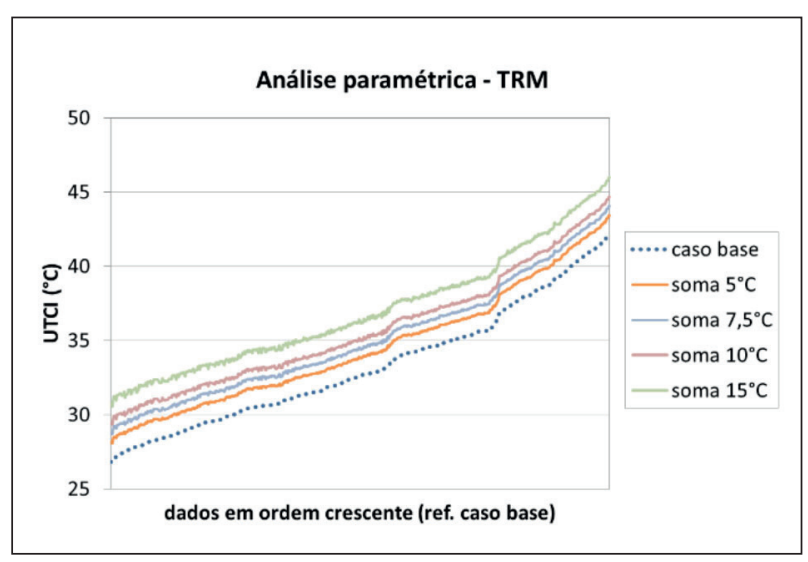

Figura 3 - Análise paramétrica do efeito isolado de alteracõoes das 4 variáveis relevantes para o cálculo do UTCl: a) Ta; b) UR; c) v; d) TRM.

Fonte: Autores. 


\section{Discussão}

Os estudos citados anteriormente mostram a tendência de diminuição de tolerância térmica para situações de desconforto térmico no caso de indivíduos, ocupantes de ambientes climatizados, relativamente àqueles que utilizam ambientes naturalmente ventilados. A título de comparação com os resultados encontrados, pode-se citar o estudo voltado a situações de calor realizado por De Vecchi et al. (2012), com usuários de prédios universitários naturalmente ventilados/ climatizados, em Florianópolis - SC. Tal estudo apontou que, para incrementos nas condições térmicas do ambiente, representadas no caso na escala SET, enquanto não se tenham notado diferenças significativas na sensação térmica entre ocupantes de ambientes naturalmente ventilados versus climatizados, os níveis de aceitabilidade térmica mostraram uma tendência claramente crescente e mais pronunciada no primeiro grupo, para aumentos graduais na escala SET. Ou seja, o estudo mostrou que o uso prévio de climatização artificial aumenta o nível de exigência térmica dos indivíduos, que corrobora os resultados obtidos.

Outro estudo de campo cujas tendências se assemelham às aqui apresentadas é o realizado por Luo et al. (2016), o qual analisa diferenças do nível de exigência térmica entre grupos de indivíduos originários de diferentes partes da China, cujo acesso a climatização artificial diferia. Tal estudo avaliou os impactos adaptativos de indivíduos que até então não tinham acesso a ambientes de qualidade térmica elevada, a esses ambientes, e a situação oposta, na qual indivíduos que utilizavam ambientes de qualidade térmica superior migravam para ambientes de qualidade inferior. Notou-se maior potencial adaptativo no primeiro grupo. Um paralelo é feito com a situação de funcionários japoneses, logo após o desastre de Fukushima, em 2011, quando o fornecimento de pico de eletricidade viu-se diminuído em 15\% (Indragantiet al., 2013). Dentre as medidas de racionamento (setsuden), estimulou-se o aumento das temperaturas de setpoint nos escritórios como medida de contenção da carga de climatização, com um mínimo recomendado para climatização artificial fixado em $28^{\circ} \mathrm{C}$. 0 estudo de campo conduzido mostrou que, embora os usuários estivessem acostumados anteriormente a ambientes com elevada qualidade térmica (leia-se controle térmico rigoroso, com baixa flutuação da temperatura interna e decorrente elevada carga de climatização), o grau de aceitabilidade térmica foi alto para as novas condições, sobretudo em virtude de medidas adaptativas. Tanabe et al. (2013) mostram, contudo, que houve uma queda de produtividade em escritórios, afetada pelas condições térmicas após as medidas de setsuden.

Yu et al. (2012) testaram o efeito do uso frequente de climatização artificial versus o não-uso (indivíduos habituados a ambientes naturalmente ventilados) em câmara climática. Verificou-se que, fisiologicamente, indivíduos habituados a ambientes climatizados têm sua capacidade de regulação fisiológica diminuída, sentindo mais calor sob situações de estresse térmico por calor ("heat shock") e reportando votos mais altos de sensação e desconforto térmicos. Para condições opostas (desconforto por frio), Yu et al. (2013) mostram que, entre dois grupos de indivíduos, de Beijing (com aquecimento artificial no inverno) e em Shanghai (sem aquecimento artificial), sob as mesmas condições de exposição ao frio em câmara climática, aqueles que em geral experimentam com maior frequência os rigores climáticos tendem a ter maior potencial adaptativo. Verificou-se uma diferença de $2-3^{\circ} \mathrm{C}$ na temperatura inferior de aceitabilidade térmica entre os grupos, sendo esta mais baixa para o grupo mais aclimatado às condições de inverno. Desta forma, verificam-se efeitos deletérios do uso intensivo de ar condicionado/aquecimento, o qual reduz até mesmo as funções fisiológicas termo-reguladoras humanas. A partir dos resultados obtidos para a amostra do Rio de Janeiro, pode-se intuir que a aclimatação no curto prazo de pessoas habituadas a ambientes climatizados seja dificultosa tanto sob o aspecto do hábito (ou histórico térmico) quanto do ponto de vista fisiológico.

Se o impacto disso em ambientes internos é o aumento na carga de climatização, em espaços externos, aumenta-se a pressão por soluções de mitigação de calor. A interdependência dos dois ambientes (interno e externo) gera também um círculo vicioso: com a intensificação por soluções de climatização artificial, gera-se mais calor para o meio externo, aumentando ainda mais o desconforto no espaço externo.

Em termos de planejamento, comparativamente à redução observada na tolerância por calor de pessoas aclimatadas (redução de $2^{\circ} \mathrm{C}$ na temperatura 
do ar, conforme a avaliação paramétrica da seção anterior), pode-se ter uma ideia do que isso representaria em termos de alterações paisagísticas. Tratando-se de cobrimento vegetal, por exemplo, Krüger e Minella (2015) mostram que a fração vegetada para áreas urbanas com 30\% de área construída poderia atingir em torno de $60 \%$ da área livre para se atingir $2^{\circ} \mathrm{C}$ de redução da temperatura ambiente (supondo-se no caso uma área de 4 hectares em Curitiba, PR). Entretanto, se a área for de grande adensamento, a fração vegetada extrapolaria os limites de área livre existente.

Para condições climáticas com predomínio de calor, o estudo de caso reportado por Barros et al. (2016), por meio de simulação computacional com o EnviMet 4, para Maceió, AL, comparou a inserção de vegetação para melhoria do microclima em situação de calor. Na comparação de alterações da vegetação do canteiro central de uma via de Maceió, verificou-se que, no horário de pico, a redução da temperatura do ar máxima encontrada entre o cenário sem vegetação para um cenário com todo o canteiro central coberto por árvores ficou abaixo de $1^{\circ} \mathrm{C}$.

Ou seja, a demanda por condições excessivamente amenas em ambientes externos exerce uma pressão por soluções urbanísticas nem sempre viáveis e, considerando a situação de pessoas aclimatadas, talvez desnecessárias, levando-se em conta seu potencial adaptativo.

\section{Conclusões}

Dos resultados apresentados observa-se que o uso prolongado de ar-condicionado impacta a percepção térmica dos usuários dos espaços públicos, mesmo quando estes não se encontram no momento em ambiente climatizado.

Deve-se reconhecer que, sob as condições de verão no Rio de Janeiro, o uso do ar-condicionado é em muitos casos inevitável, sendo uma das estratégias indicadas para redução do desconforto por calor para essa cidade. Entretanto, o setpoint adotado para a temperatura operativa de ambientes climatizados deveria ser mais bem compreendido e especificado, uma vez que é essa temperatura que influirá no histórico e percepção térmica do usuário. Nesse sentido, a operação de equipamentos de climatização com maior atenção às condições climáticas vigentes, suavizaria o gradiente térmico entre clima interno e externo às edificações, trazendo uma menor pressão dos usuários dos espaços externos por alterações no desenho urbano.

A partir dos resultados encontrados, recomendamos para o desenvolvimento da área de conforto em espaços abertos no Brasil (cuja maior parte do território apresenta média das temperaturas máximas anuais semelhante ou até mesmo superior às da cidade do Rio de Janeiro e onde o uso de ar-condicionado é frequente), no que tange a levantamentos de percepção térmica junto à população, a introdução do questionamento acerca do uso de ar-condicionado no levantamento de campo. 0 tratamento dos dados, diante dos resultados apresentados no presente estudo, deve ser realizado a partir disso, por grupos claramente diferenciados por uso ou não da climatização artificial.

\section{Referências}

American Society of Heating, Refrigerating; AirConditioning Engineers - ASHRAE (2004). Standard 55: thermal environmental conditions for human occupancy. Atlanta, USA.

Arens, E., Humphreys, M. A., De Dear, R., \& Zhang, H. (2010). Are 'class A' temperature requirements realistic or desirable? Building and Environment, 45(1),4-10.

Barbosa, G. S., Drach, P. R. C., \& Corbella, O. D. (2010a). A Comparative Study of Sprawling and Compact Areas in Hot and Cold Regions. In Proceedings o the $11^{\text {th }}$ World Renewable Energy Congress. Abu Dhabi.

Barbosa, G. S., Drach, P. R. C., \& Corbella, O. D. (2010b). Um Estudo Comparativo de Regiões Espraiadas e Compactas: caminho para o desenvolvimento de cidades sustentáveis. In Anais do Congresso Internacional Sustentabilidade $e$ Habitação de Interesse Social. Porto Alegre, RS.

Barros, C. P. G., Costa, S. B., \& Barbosa, R. V. R. (2016). Vegetação e microclima urbano: estudo de caso em ruas residenciais de bairros litorâneos em Maceió-Alagoas. In Anais do $7^{\circ}$ Congresso Luso-Brasileiro para o Planejamento Urbano, Regional, Integrado e Sustentável.Maceió, AL.

BioKlima v. 2.6 (software) (2016). Recuperado de https:// www.igipz.pan.pl/Bioklima-zgik.html 
Bröde, P., Fiala, D., Błażejczyk, K., Holmér, I., Jendritzky, G., Kampmann, B., Tinz, B., \& Havenith, G. (2012). Deriving the Operational Procedure for the Universal Thermal Climate Index UTCI. International Journal of Biometeorology, 56(3), 481-494.

Busch, J. F. (1992). A Tale of Two Populations: thermal comfort in air-conditioned and naturally ventilated offices in Thailand. Energy and Buildings, 18(3), 235-249.

Cândido, C. M., De Dear, R., Lamberts, R., \& Bittencourt, L. (2010). Cooling exposure in hot humid climates: are occupants "addicted"? Architectural Science Review, 53, 59-64.

Chen, L., \& Ng, E. (2012). Outdoor thermal comfort and outdoor activities: A review of research in the past decade. Cities, 29, 118-125.

Chun, C., Kwok, A., Mitamura, T., Miwa, N., \& Tamura, A. (2008). Thermal Diary: connecting temperature history to indoor comfort. Building and Environment, 43(5), 877-885.

De Dear, R., \& Fountain, M. E. (1994). Cover Feature: thermal comfort in air-conditioned office buildings in the tropics. Journal of the Australian Institute of Refrigerating, AirConditioning and Heating,48(9), 14-30.

De Dear, R., Kim, J., Candido, C., \& Deuble, M. (2014). Summertime Thermal Comfort in Australian School Classrooms. In Proceedings of the $8^{\text {th }}$ Windsor Conference. Cumberland Lodge, Windsor UK.

Deuble, M. P., \& De Dear, R. (2012). Mixed-Mode Buildings: a double standard in occupants' comfort expectations Related. Building and Environment, 54(8), 53-60.

De Vecchi, R., Cândido, C., \& Lamberts, R. (2012). Thermal history and its influence on occupants' thermal acceptability and cooling preferences in warm humid climates: A new desire for comfort. In Proceedings of the 7th Windsor Conference. Cumberland Lodge, Windsor UK.

Drach, P. R. C., \& Corbella, O. D. (2010). Estudo das Alterações na Dinâmica da Ventilação e da Temperatura na Região Central do Rio de Janeiro: mudanças na ocupação do solo urbano. In Anais do 4º Congresso Luso-Brasileiro para o Planejamento Urbano, Regional, Integrado e Sustentável (PLURIS 2010).Faro, Portugal.

Fadeyi, M. O. (2014). Initial Study on the Impact of Thermal History on Building Occupants' Thermal Assessments in Actual Air-Conditioned Office Buildings. Building and Environment, 80, 36-47.
Fiala, D., Havenith, G., Bröde, P., Kampmann, B., \& Jendritzky, G. (2012). UTCI - Fiala Multi-Node Model of Human Heat Transfer and Temperature Regulation. International Journal of Biometeorology, 56(3), 429-441.

Indraganti, M., Ooka, R., \& Rijal, H. (2013). Thermal comfort in offices in summer: findings from a field study under the 'setsuden' conditions in Tokyo, Japan, Building and Environment, 61, 114-132.

International Organization for Standardization - ISO (1995). ISO 10551: ergonomics of the thermal environment: assessment of the influence of the thermal environment using subjective judgment scales. Genebra.

International Organization for Standardization - ISO (1998). ISO 7726: ergonomics of the thermal environment: instruments for measuring physical quantities. Genebra.

International Organization for Standardization - ISO (2007). ISO 9920: ergonomics of the thermal environment: estimation of the thermal insulation and evaporative resistance of a clothing ensemble. Genebra.

Johansson, E., Thorsson, S., Emmanuel, R., Krüger, E. (2014). Instruments and Methods in Outdoor Thermal Comfort Studies: the need for standardization. Urban Climate, 10, 346-366.

Krüger, E., Drach, P., Emmanuel, R., \& Corbella, O. (2012). Estudo de Conforto em Espaços Abertos em Região de Clima Temperado: o caso de Glasgow, Reino Unido. Ambiente Construído, Porto Alegre, 12(1), 7-25.

Krüger, E., \& Minella, F. (2015).Proposition of an index for quantifying the amount of vegetated fraction needed for air temperature changes in urban locations. Proceedings of the 9th International Conference on Urban Climate. Toulouse, França.

Lin, C., Lin, T. P., \& Hwang, R. L. (2013). Thermal Comfort for Urban Parks in Subtropics: understanding visitor's perceptions, behavior and attendance. Advances in Meteorology, v. 2013. Recuperado em 01 Jan. 2017 de http://dx.doi. org/10.1155/2013/640473

Luo, M., De Dear, R., Ji, W., Bin, C., Lin, B., Ouyang, Q., \& Zhu, Y. (2016). The dynamics of thermal comfort expectations: the problem, challenge and implication. Building and Environment, 95, 322-329.

Monteiro, L. M., \& Alucci, M. P. (2012). Modelo Adaptativo de Conforto Para Avaliação in loco de Espaços Urbanos Abertos. Ambiente Construído, Porto Alegre, 12(1), 61-79. 
Ndetto, E. L., \& Matzarakis, A. (2013). Basic Analysis of Climate and Urban Bioclimate of Dar es Salaam, Tanzania. Theoretical and Applied Climatology, 114(1/2), 213-226.

Ndetto, E. L., \& Matzarakis, A. (2015). Urban Atmospheric Environment and Human Biometeorological Studies in Dar es Salaam, Tanzania. Air Quality, Atmosphere \& Health, 8(2), 175-191.

Nince, P. C., Musis, C., Biudes, M., Nogueira, J., \& Nogueira, M. (2013). Usos dos Índices PET e UTCI na Avaliação do Conforto Termal no Campus da UFMT em CuiabáMT. Revista Eletrônica em Gestão, Educação e Tecnologia Ambiental, 9(9), 2026-2036.

Peel, M. C., Finlayson, B. L., \& Mcmahon, T. A. (2007). Updated World Map of the Köppen-Geiger Climate Classification. Hydrology and Earth System Sciences Discussions, 4(2), 439-473.

Rossi, F. A., Krüger, E. L., \& Bröde, P. (2012). Definição de Faixas de Conforto e Desconforto Térmico Para Espaços Abertos em Curitiba, PR, com o Índice UTCI. Ambiente Construído, Porto Alegre, 12(1), 41-59.
Tanabe, S., Iwahashi, Y., Tsushima, S., \& Nishihara, N. (2013). Thermal comfort and productivity in offices under mandatory electricity savings after the Great East Japan earthquake. Architectural Science Review, 56(1), 4-13.

Yu, J., Ouyang, Q., Zhu, Y., Shen, H., Cao, G., \& Cui, W. (2012). A comparison of the thermal adaptability of people accustomed to air-conditioned environments and naturally ventilated environments. Indoor air, 22(2), 110-118.

Yu, J., Cao, G., Cui, W., Ouyang, Q., \& Zhu, Y. (2013). People who live in a cold climate: thermal adaptation differences based on availability of heating. Indoor air, 23(4), 303-310.

Recebido: Nov. 08, 2016

Aprovado: Jan. 05, 2017 